\title{
On the Composition of Simultaneous Differential Systems of the First Order
}

By M. Mursi-Ahmed, St. John's College, Cambridge.

(Received 17th March, 1932. Read 6th May, 1932.)

$\S 1$. Consider the system of $n$ first order linear differential equations:

$$
\frac{d u_{i}}{d x}+\sum_{k=1}^{n} g_{i k}(x) u_{k}=0 \quad(i=1,2, \ldots n),
$$

together with the $n$ boundary conditions

$$
\sum_{k=1}^{n} a_{i k} u_{k}(a)+\sum_{k=1}^{n} b_{i k} u_{k}(b)=0 \quad(i=1,2, \ldots n),
$$

where $a_{i j}, b_{i j}$ are constants and where we assume for simplicity of notation that $g_{i i} \equiv 0$.

We write this system of equations and boundary conditions in matrix notation thus:

$$
\begin{aligned}
& {\left[\begin{array}{llll}
d / d x & g_{12} & \ldots & g_{1 n} \\
g_{21} & d / d x & \ldots & g_{2 n} \\
\ldots & \ldots & \ldots & \ldots \\
g_{n 1} & g_{n 2} & \ldots & d / d x
\end{array}\right]\left[\begin{array}{c}
u_{1} \\
u_{2} \\
\cdots \\
u_{n}
\end{array}\right]=0} \\
& {\left[\begin{array}{cccc}
a_{11} & a_{12} & \ldots & a_{1 n} \\
a_{21} & a_{22} & \ldots & a_{2 n} \\
\ldots & \ldots & \ldots & \ldots \\
a_{n 1} & a_{n 2} & \ldots & a_{n n}
\end{array}\right]\left[\begin{array}{c}
u_{1}(a) \\
u_{2}(a) \\
\ldots \ldots \\
u_{n}(a)
\end{array}\right]+\left[\begin{array}{cccc}
b_{11} & b_{12} & \ldots & b_{1 n} \\
b_{21} & b_{22} & \ldots & b_{2 n} \\
\ldots \ldots & \ldots & \ldots & \ldots \\
b_{n 1} & b_{n 2} & \ldots & b_{n n}
\end{array}\right]\left[\begin{array}{c}
u_{1}(b) \\
u_{2}(b) \\
\ldots . \\
u_{n}(b)
\end{array}\right]=0,}
\end{aligned}
$$

or with an obvious notation

$$
\begin{gathered}
P U=0 \\
W_{a} U(a)+W_{b} U(b)=0 .
\end{gathered}
$$

It is the product of operator systems of the form

$$
\begin{gathered}
P \\
W_{a}+W_{b}
\end{gathered}
$$

and

$$
\begin{gathered}
Q \\
Z_{a}+Z_{b}
\end{gathered}
$$

that will be considered in this paper. It will be shown in $\S 2$ that if we define the product of two such systems in the same manner as 
J. M. Whittaker ${ }^{1}$ defined it in the case $n=1$, then the Green's matrix of the product system is the product by composition of the second kind of the Green's matrices of the component systems. In $\S 3$ the product of a system into its adjoint system will be considered and it will be shown that the differential equations of the second order which appear in the product system can all be obtained from a quadratic form in the elements of the component systems in the same way that the Lagrange's equations in dynamics are obtained from the energy function. Lastly a relation is obtained between the solution of the non-homogeneous product system and the solutions of the component systems, when $n=1$ and the order is no longer restricted to be the first.

$\S 2$. The Green's matrix for the system (1) is defined by Birkhoff ${ }^{2}$ to be the matrix

$$
G(x, \xi) \equiv\left[\begin{array}{c}
G_{11}(x, \xi), \ldots, G_{1 n}(x, \xi) \\
G_{21}(x, \xi), \ldots, G_{2 n}(x, \xi) \\
\ldots \ldots, \ldots, \ldots, \ldots, G_{m}(x, \xi)
\end{array}\right],
$$

where $G_{i j}(x, \xi), i \neq j$ are continuous functions with continuous first derivatives, while $G_{i i}(x, \xi)$ each have only one discontinuity, a jump of amount 1 , at $x=\xi$, i.e.

$$
G(x, x-0)-G(x, x+0)=I,
$$

where $I$ is the unit matrix. Further, each column of the above matrix satisfies the system of equations except at $x=\xi$, and satisfies the boundary conditions identically in $\xi$.

Let $L(x, \xi), M(x, \xi)$ be the Green's matrices thus defined for the systems (2) and (3) respectively; then the Green's function of

$$
\begin{aligned}
& P Q U=0, \\
& Z_{\imath} U(a)+Z_{b} U(b)=0, \\
& {\left[W_{a} Q\right] U(a)+\left[W_{b}, Q\right] C(b)=0,}
\end{aligned}
$$

is given by

$$
G(\dot{x}, \xi)=\int_{a}^{b} M(x, \eta) L(\eta, \xi) d \eta \text {. }
$$

For since

$$
G_{i j}(x, \hat{\xi})=\int_{a}^{b} \sum_{k=1}^{n} M_{i k}(x, \eta) L_{k j}(\eta, \hat{\xi}) d \eta
$$

1 Proc. Edin. Math. Soc., 3 (1932), 10; Dr Whittaker considers the case when the operator is of any order.

${ }^{2}$ Birkhoff and Langer, Proc. Amer. Acad. Arts d. Sc, 58 (192:3), 59. 
we see that $G_{i j}(x, \xi)$ is continuous. It is also seen from the nature of the discontinuities of $L_{i k}(x, \xi)$ and $M_{i k}(x, \xi)$ that $G_{i ;}$ has a continuous first derivative unless $x=\xi$ where $G_{i i}$ has a jump of magnitude unity. Further,

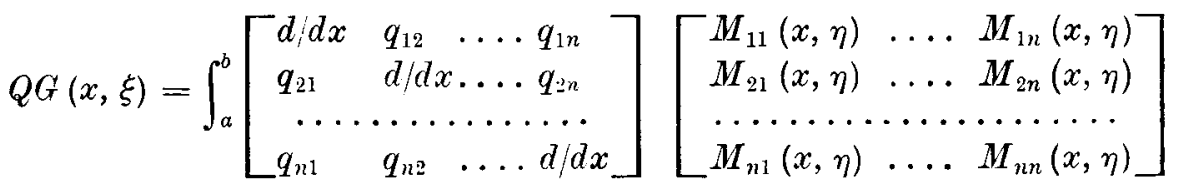

$$
\begin{aligned}
& \times\left[\begin{array}{ccc}
L_{11}(\eta, \xi) & \ldots & L_{1 n}(\eta, \xi) \\
L_{21}(\eta, \xi) & \ldots & L_{2 n}(\eta, \xi) \\
\ldots \ldots & \ldots & \ldots \\
L_{n 1}(\eta, \xi) & \ldots & L_{n n}(\eta, \xi)
\end{array}\right] d \eta \\
& =\left[\begin{array}{lll}
L_{11}(x, \xi) & \ldots & L_{1 n}(x, \xi) \\
\ldots & \ldots & \ldots \\
L_{n 1}(x, \xi) & \ldots & L_{n n}(x, \xi)
\end{array}\right],
\end{aligned}
$$

since each column of $M(x, \xi)$ satisfies the system $Q$, the only discontinuities being along the principal diagonal; hence

$$
P Q G(x, \hat{\xi})=0,
$$

and $G(x, \hat{\xi})$ satisfies the boundary conditions of $(4)$, and so is the Green's matrix for the product system.

$\S 3$. The adjoint to the system (1) is given by ${ }^{1}$

or

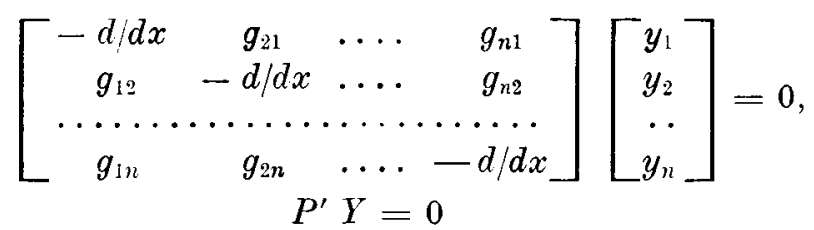

together with the boundary conditions

$$
\left[y_{1}(a) \ldots y_{n}(a)\right] W_{a}^{-1}+\left[y_{1}(b) \ldots y_{n}(b)\right] W_{b}^{-1}=0 .
$$

Then we state the following result which can easily be verified: let

$$
Q(u)=\sum_{k=1}^{n} u_{k}^{\prime 2}+\sum_{i, r, k=1}^{n} g_{i k} g_{r k} u_{i} u_{r}-\sum_{i, r=1}^{n} g_{r i} u_{i}^{\prime} u_{r}+\sum_{i, k=1}^{n} g_{i k}^{2} u_{i}^{2},
$$

where $g_{s s}=0$ and in the second summation $i \neq r$; then the differential equations of the system

$$
P P^{\prime} Y=0
$$

1 Birkhoff and Langer, loc. cit., 64. 
are given by

$$
\frac{d}{d x}\left\{\frac{\partial Q}{\left.\partial u_{i}^{\prime}\right\}}-\frac{\partial Q}{\partial u_{i}}=0 \quad(i=1,2, \ldots n) .\right.
$$

This incidentally proves that this system is self-adjoint. ${ }^{1}$

$\S 4 . \quad$ Let

$$
\begin{aligned}
P y_{1} & =0 \\
{\left[A_{i} y_{1}\right]_{a} } & +\left[B_{i} y_{1}\right]_{b}=0 \quad(i=1,2 \ldots p),
\end{aligned}
$$

where $P$ is a linear operator of order $p$, be an incompatible system. The solution of

$$
\begin{aligned}
Q z & =f(x) \\
{\left[C_{i} z\right]_{a}+\left[D_{i} z\right]_{b}=0 } & (i=1,2, \ldots 2),
\end{aligned}
$$

when the corresponding homogeneous system is incompatible, is given by

$$
z(x)=\int_{a}^{b} M(x, \xi) f(\xi) d \xi
$$

Then we state the following result: the solution of the product system

is given by

$$
\begin{aligned}
& P Q u(x)=f(x) \\
& {\left[A_{i} Q u\right]_{a}+\left[B_{i} Q u\right]_{b}=0} \\
& {\left[C_{i} u\right]_{a}+\left[D_{i} u\right]_{b}=0}
\end{aligned}
$$

$$
u(x)=\int_{a}^{b} M(x, \eta) y_{1}(\eta) d \eta
$$

in terms of solutions of the component systems; where

$$
y_{1}(x)=\int_{a}^{b} L(x, \zeta) f(\zeta) d \zeta
$$

is the solution of the system

$$
\begin{aligned}
& P y_{1}=f(x) \\
& {\left[A_{i} y_{1}\right]_{a}+\left[B_{i} y_{1}\right]_{b}=0 \quad(i=1,2, \ldots, p) .}
\end{aligned}
$$

${ }^{1}$ See Hilbert, Gött. N(ch. (1906), 474. 